\title{
Droplet generation in ensembles of randomly corrugated ligaments
}

\author{
Sagar Pal ${ }^{1}$, Marco Crialesi-Esposito ${ }^{2}$, Daniel Fuster ${ }^{1}$ and Stephane Zaleski ${ }^{13^{*}}$ \\ IInstitut Jean le Rond J'Alembert, Sorbonne Université and CNRS, Paris, France \\ 2Department of Engineering Mechanics,Royal Institute of Technology (KTH), Stockholm, Sweden \\ ${ }^{3}$ Institut Universitaire de France (IUF), Paris, France
}

*Corresponding author email: stephane.zaleski@sorbonne-universite.fr

\begin{abstract}
This study focuses on a statistical description of droplet sizes, created as a result of capillaryinduced breakup of ligaments. Direct numerical simulations of air-water systems are employed by solving the two-phase Navier-Stokes equations on adaptive Octree grids ( $h$ ttp://basilisk.fr/), using the VOF methodology coupled with height-function-based curvature modeling. Breakup of individual ligaments are triggered by initial surface corrugations, the dynamics of which are deterministic. Stochasticity is introduced in the mix by conducting an ensemble of simulations of slender corrugated ligaments, each realization corresponding to a random but unique initial configuration. Probability density functions of the droplet sizes are computed for different ensemble sizes. These results (fig. 1) combining the effects of stochasticity with the capillarity-driven nonlinear dynamics are compared to the predictions of a Gaussian random process theory for nearmonochromatic waves, which facilitate our understanding of the nature of drop size distributions encountered in realistic and convoluted fluid fragmentation scenarios.
\end{abstract}

\section{Keywords}

drop size distributions, atomization, DNS of air-water systems, ligaments, Gamma distribution.

\section{Introduction}

The determination of the distribution of droplet sizes is one of the central problems in the study of atomization itself a key problem in many respects [2]. Despite a long history of study, it is still difficult to determine the distribution and to sort out the mechanisms that lead to it. From the point of view of elementary deterministic processes in Fluid Mechanics, it is possible to distinguish four stages in the atomization process

- The development of a primary instability, often the Kelvin-Helmholtz instability, in which modes that have the highest growth rate are most amplified [4]. The instability leads to the formation and growth of waves and vortical structures on the interface.

- The waves grow and stretch sufficiently to form thin sheets and Taylor-Culick end rims $[15,9]$. In the process of growing and stretching the sheets acquire curvature similar to the bag mode of droplet atomization [13]. 
- The waves perforate at so-called weak spots $[11,16]$ and the resulting holes expand with additional end rims that may shed droplets. Eventually the expansion stops and leaves a network of ligaments.

- The ligaments break into droplets.

Another point of view on the atomization process emphasizes the interaction between the liquid and the high-speed surrounding air. This interaction operates in two stages

1. In the first stage of the air dynamic turbulent evolution, large scale turbulent vortical structures form, such as the Kelvin-Helmholtz billows. This first stage is a kind of inverse cascade or two-dimensional turbulent process, in which vortical structures merge into larger ones (vortex pairing). The waves and sheets are sucked or engulfed into the large billows and reach a larger outer length scale $L_{0}$.

2. In the second stage a direct three dimensional cascade ensues, with a Reynolds number and dissipation based on the outer length scale $L_{0}$.

These two processes lead, in a manner that is not yet fully understood, to a final distribution of droplet sizes. This distribution has often been considered to be Log-Normal, although other distributions are also suggested (Pareto [2], Weibull, Rosin-Rammler, Gamma etc.). The LogNormal distribution takes the form

$$
P_{e}(d)=\frac{1}{\hat{\sigma} d \sqrt{2 \pi}} \exp \left[-\frac{(\ln d-\hat{\mu})^{2}}{2 \hat{\sigma}^{2}}\right],
$$

Where $d$ is the droplet size, $P_{e}(d) \mathrm{d} d$ is the fraction of the total number of droplets between $d$ and $d+\mathrm{d} d, \hat{\sigma}$ is the Geometric Standard Deviation and $\hat{\mu}$ is the geometric average.

The Log-Normal distribution has been derived by Kolmogorov [8] for the problem of fragmentation of coal particles and further developed by Gorokhovski and Saveliev $[5,6$.] The mechanism proposed by Kolmogorov is not easy to transpose to atomization since the direct fragmentation of a droplet into smaller daughter droplets is opposed by surface tension forces below the Hinze scale, which is usually much larger than the observed droplets. Instead, the multistep fragmentation process above is actually occurring, leading in the final stage to rim and ligaments whose diameter is already below the Hinze scale.

While the mechanisms that lead to the particle size distribution in the full case are extremely complex and involve in particular the multiple manners in which a thin sheet may break $[11,16]$ the final stage, ligament breakup, can be modelled by requiring only the macroscopic, NavierStokes fluid equations with sharp interfaces and surface tension. In other words, the full problem, and in particular the problem of sheet breakup, is ill-posed. By ill-posed we mean that additional physical descriptions or mechanisms, such as nanoscale physics or hypotheses about heterogeneities have to be made to understand sheet breakup and perforation. On the other hand the ligament breakup problem is markedly simpler.

\section{Methods}

The Navier-Stokes equations with surface tensions and two phases are solved using a finite volume formulation on an octree grid [14]. The two phases are liquid and gas, and in the absence of significant gas flow, the gas phase, similar to air, does not play a role in the dynamics. Surface tension is modelled using the height function method and a well-balanced force formulation [14]. Viscosity is dealt with in an implicit manner and the Bell-Collela-Glaz scheme, coupled with two projection steps, is used for velocity advection and pressure. The whole scheme is coded in the BasiliskC language with a source available freely on the platform http://basilisk.fr . The liquid phase is initially at rest with a random shape consisting of a 
slightly perturbed or corrugated constant-diameter cylinder, that is if $x$ is the coordinate along the axis of the cylinder, then the local cylinder diameter or width obeys

$$
r(x)=r_{0}+\epsilon(x)
$$

where $\varepsilon$ is a nearly Gaussian noise and $r_{0}$ is the mean radius. The amplitude of the perturbation is $\epsilon_{0}=\left\langle\epsilon^{2}\right\rangle^{1 / 2}$ and its dimensionless measure is $\eta=\varepsilon_{0} / r_{0}$. Assuming a similar setup Villermaux has claimed the final distribution is a Gamma distribution described by

$$
P_{\Gamma}(d ; \alpha, \beta)=\frac{\beta^{\alpha}}{\Gamma(\alpha)} d^{\alpha-1} \exp (-\beta d)
$$

where the exponent $\alpha$ that describes the shape of the distribution is related to the initial corrugation and the parameter $\beta$ is related to the mean droplet diameter $\langle d\rangle$.

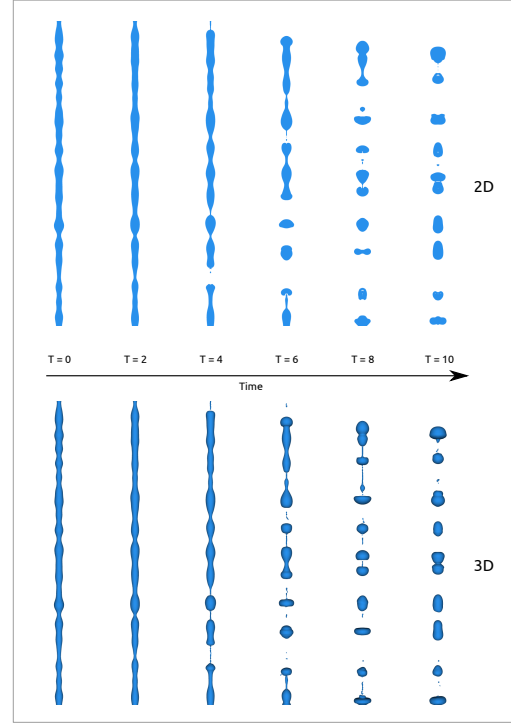

(a)

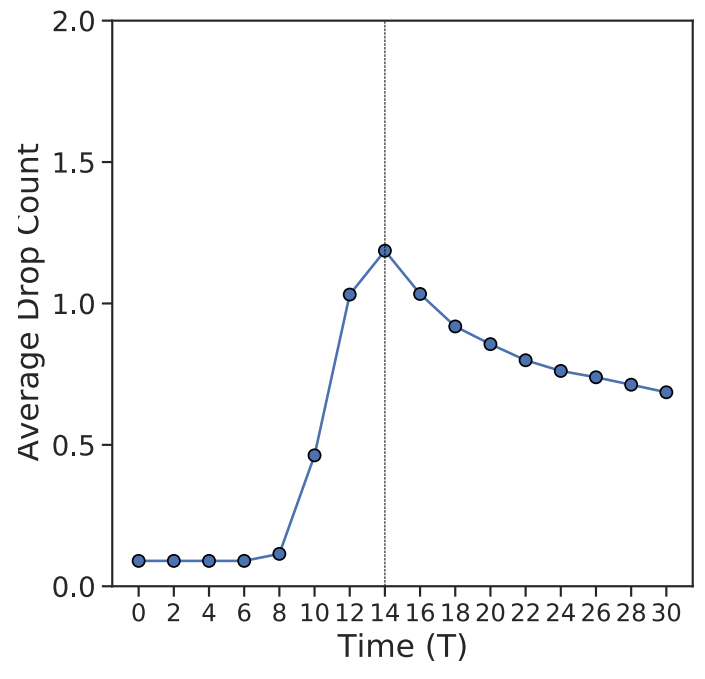

(b)

Figure 1: (a) 2D versus 3D computations with the same initial conditions. The results are close, and the distribution of droplet sizes is near-identical. (b) Average drop count as a function of time. The maximum drop count is reached at $T=14$ which is close to the time $\mathrm{T}=12$ at which the subsequent analysis is performed. This time is long enough so that most breakups have occurred, but few coalescences of drops occurred.

We also try to fit with the Gaussian distribution

$$
P_{G}(d)=\frac{1}{\sigma_{1} d \sqrt{2 \pi}} \exp \left[-\frac{\left(d-\mu_{1}\right)^{2}}{2 \sigma_{1}^{2}}\right]
$$

where $\sigma_{!}$is the standard deviation and $\mu_{1}$ is the average.

We here report results obtained with the dimensionless initial radius $r_{0}$ corresponding to the Ohnesorge number $\mathrm{Oh}=\mu /\left(\sigma r_{0} \rho\right)^{1 / 2}=10^{-3}$. The aspect ratio $\Lambda=\mathrm{L} / r_{0}$ is 100 (where $\mathrm{L}$ is the periodicity length along $x$ ). For water this corresponds to an approximate radius of $1 \mathrm{~cm}$. Both full 3D and axisymmetric simulations are performed and are in good agreement with each other (Figure 1a). Initially one sees a large number of breakups, while after some time breakups 
become less frequent and coalescence more frequent. As shown on Figure 1b, breakups and coalescence balance around $T=14$. The axisymmetric and periodic setup forces droplet to remain on a single axis and promotes coalescence in an unrealistic manner. In order to have a better comparison with experiments, we select time $T=12$ to measure the probability

distributions, since it has a smaller amount of unrealistic coalescence. The results are show on Figure $2 \mathrm{a}$ and $2 \mathrm{~b}$. The leftmost peak in $2 \mathrm{a}$ corresponds to secondary or "satellite" droplets that form from the liquid bridges joining to main droplets formed at the Rayleigh Plateau wavelength. The peak at $\mathrm{d} / 2 \mathrm{r}_{0}=1.9$ corresponds to one Rayleigh-Plateau wavelength: taking a ligament segment length close to the optimal RP wavelength $I=9 r_{0}$ one obtains $d=\left(3 \mathrm{l} / 4 r_{0}\right)^{1 / 3}$ $=1.88$ and the peak at $\mathrm{d} / 2 \mathrm{r}_{0}=2.3$ corresponds to two such wavelengths. When one zooms on the tail in Fig $2 \mathrm{~B}$ pf the count in log-lin coordinates an additional pseudo-periodicity is seen, and the droplet count abruptly falls to zero after after a dimensionless critical diameter $d_{c} / 2 r_{0} \sim 4.3$.

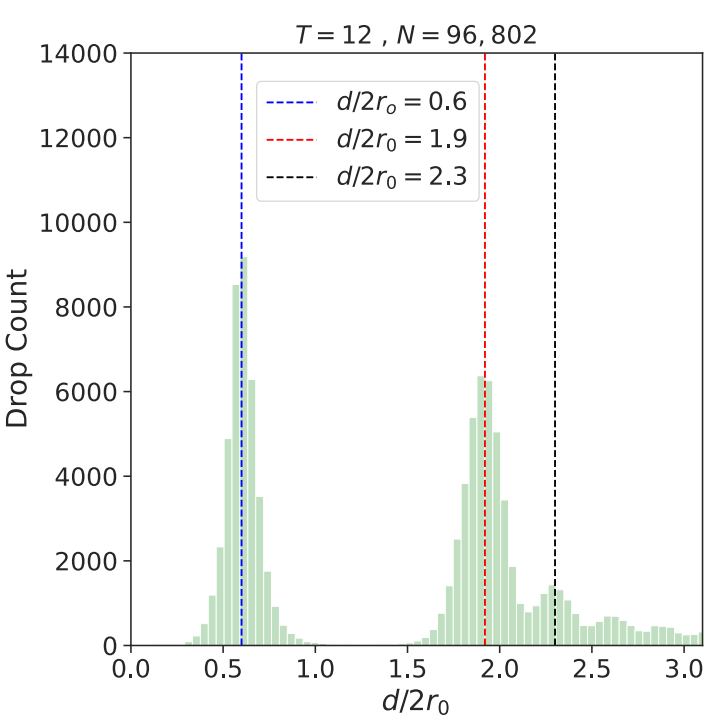

(a)

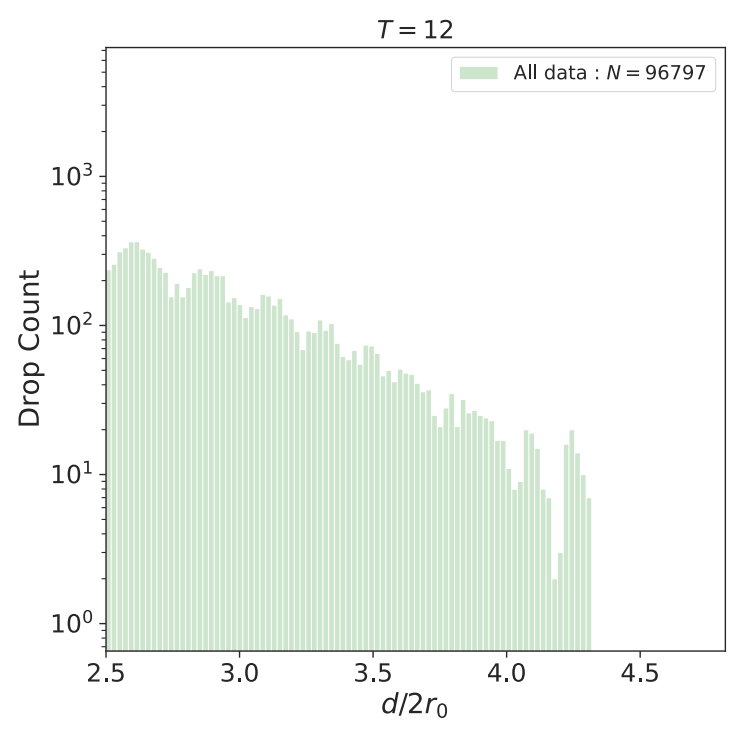

(b)

Figure 2: (a) Droplet count at $\mathrm{T}=12$ in linear coordinates. The leftmost peak corresponds to secondary or "satellite" droplets that form from the liquid bridges joining to main droplets formed at the Rayleigh Plateau wavelength. The peak at $\mathrm{d} / 2 \mathrm{r}_{0}=1.9$ corresponds to one Rayleigh-Plateau wavelength and the peak at $\mathrm{d} / 2 \mathrm{r}_{0}=2.3$ corresponds to two such wavelengths (b) A zoomed view of the tail of the count in loglin coordinates.

The distributions are obtained by averaging a large number of numerical experiments on independent periodic ligament simulations that yield 96695 total drops. The statistical error in the distribution is estimated by constructing several ensembles from the largest 96695 total drops super-ensemble and then estimating inter-ensemble variability. We note that for such large ensembles the estimated statistical error, plotted as an error bar in Figure 3, is very small. Figure 3 offers another view of the distribution than Figure 2a: there, the ordinate is plotted on a logarithmic scale, and the bins are wider which erases the oscillations seen in Figure $2 b$ and emphasizes the linear decay of the logarithm of the frequency, that is an exponential decay. To fit the distribution, we perform some filtering operations

1. Select a range of data that excludes the region of the satellite droplets, since these are strongly time dependent, disappearing more or less rapidly with coalescence with the 
larger droplets. We not that a specific "bump" for satellite droplet sizes has not been observed in experiments.

2. Exclude the large droplet sizes that have zero counts.

We attempt to fit the Normal or Gaussian, Log-Normal law and Gamma described above (eqs 1-3).

Other potential forms such as Pareto [2], Poisson and volume-based Poisson have also been tried. The equations are

$$
P_{P}(d)=\lambda \exp (-\lambda d)
$$

for the Poisson distribution of rate $\lambda$

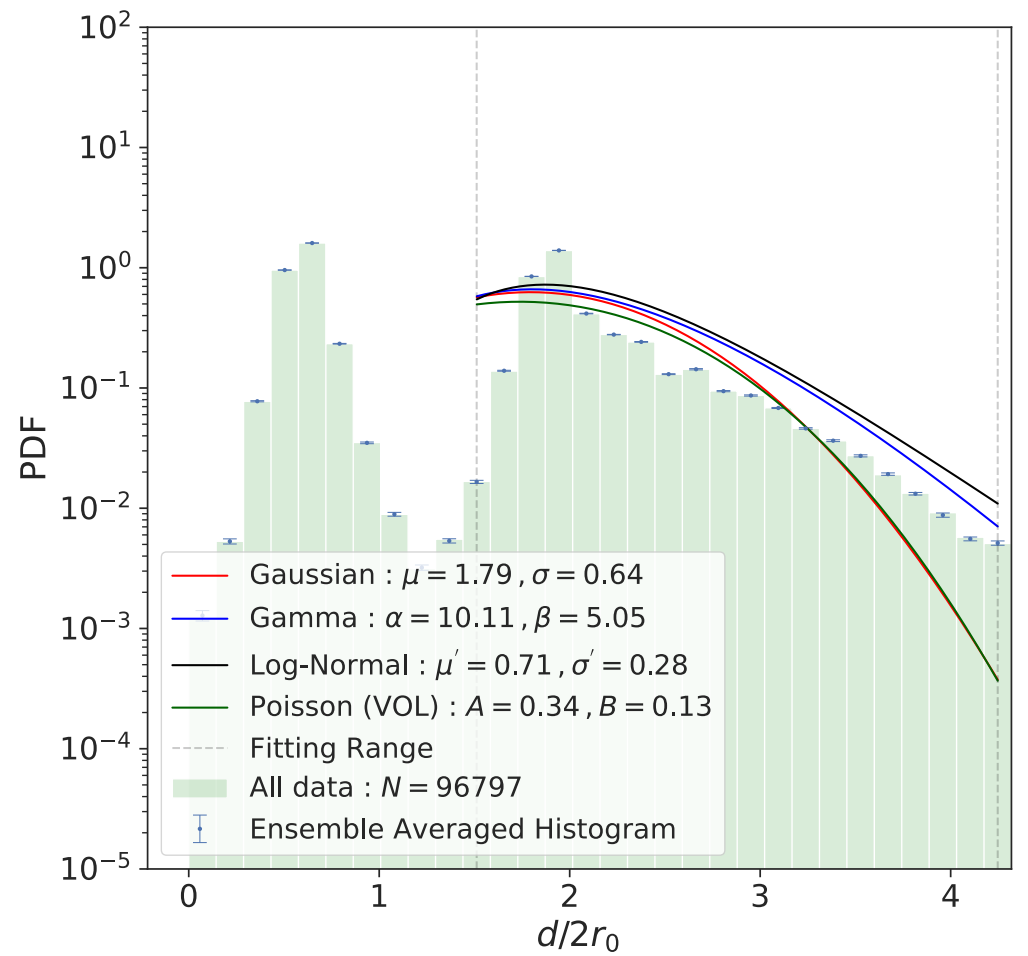

Figure 3: A log-linear plot in with a fit to the Log-Normal distribution (eq. 1) the Gamma distribution (eq. 2), the Gaussian (eq. 3) and the volume-based Poisson (eq. 5).

A volume-based Poisson distribution of rate $\lambda$ is obtained in the following way: consider the initial cylinder of fluid, and that it is cut randomly into a number of segments of lengths $I_{1}, I_{2}, \ldots$ $I_{n}$. Each segment has volume $\pi l_{i} r_{0}^{2}$. The equivalent droplet size of this segment is thus given by

$$
\pi d^{3} / 6=\pi l_{i} r_{0}^{2}
$$

and thus, the distribution of ligament segment lengths $\mathrm{P}^{\prime}$ is related to the distribution of droplet sizes $P(d)$ by 


$$
d^{2} P^{\prime}\left(d^{3}\right)=6 r_{0}^{2} P(d)
$$

If the ligament segment lengths are given by a Poisson distribution (eq. 4) then the droplets are distributed by a "volume-based" Poisson distribution

$$
P_{V P}^{\prime}(d)=3 \lambda^{\prime} d^{2} \exp \left(-\lambda^{\prime} d^{3}\right) .
$$

Finally, a distribution that was found to work well for aerosols produced by sneezes is the Pareto distribution

$$
P_{P a}(d)=C d^{-\alpha}
$$

where $\alpha$ is a parameter characterizing the Pareto distribution and $\mathrm{C}$ is a normalization constant depending on the range in which the Pareto distribution is fitted. If the range is $\left[d_{1}, d_{2}\right]$ then

$$
C=\left(d_{1}^{-\alpha+1}-d_{2}^{-\alpha+1}\right)^{-1}
$$

it is useful to note that a similar normalization constant should be added to the distributions (eqs 1-5) whenever they are fitted over a limited range.

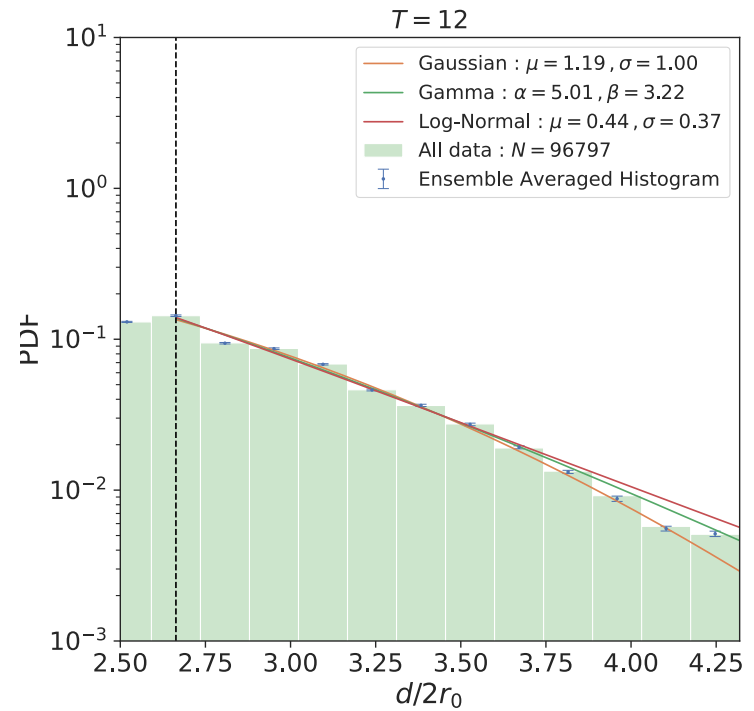

(a)

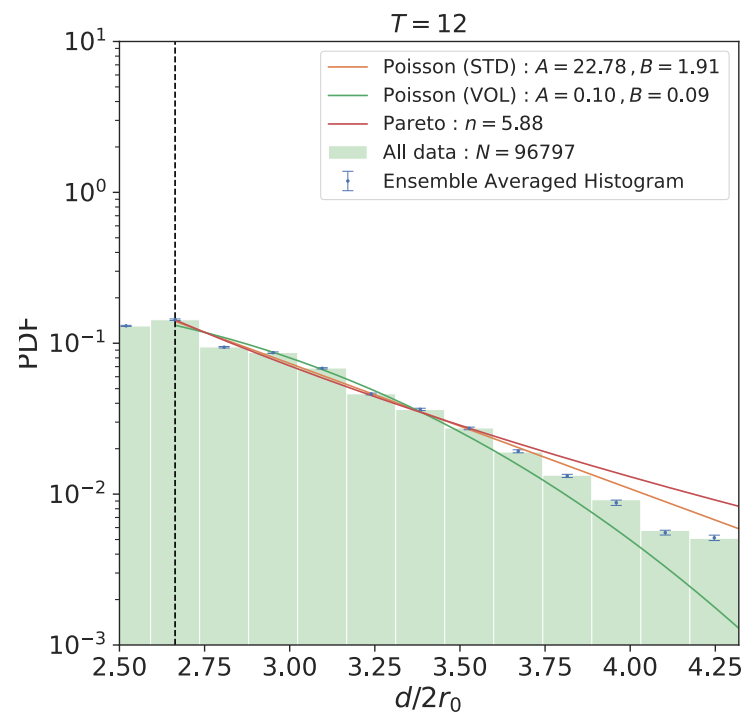

(b)

Figure 4: A zoomed view of the large-diameter part of the distribution. (a): fits to the two parameter functional forms: Gaussian, Log Normal and Gamma fits (b) fits to the single parameter functional forms Pareto, standard Poisson and volume-based Poisson.

In Figure 4, in order to elucidate which functional form fits best the distribution of large droplets, we fit tail of the distribution defined by $3.1<d /\left(2 r_{0}\right)<4.3$. In Figure $4 a$, we fit the two parameter distributions (eqs 1-3): the Gaussian form fits all the bins within the error bars except the last one. The Log-Normal form fits all the bins except the penultimate one. The Gamma form is between the previous two and is in that sense the "best" fit. In Figure 4b we fit the oneparameter distributions: Pareto, Poisson and volume-based Poisson (4-6). The one parameter distributions perform worse than the two-parameter ones but a smaller number of parameters is desirable when extracting asymptotic behavior. 
All the forms above fail to describe the total dearth of droplets with relative diameter above 4.3. An alternate distribution would be

$$
P_{P L}(d)=\left\{\begin{array}{cc}
C^{\prime} \exp (-\lambda d) & \text { if } d \leq d_{c} \\
0 & \text { if } d>d_{c}
\end{array}\right.
$$

where $C^{\prime}$ is a normalization constant depending on $\lambda$ and $d_{c}$. A similar modification of the other distributions could be considered. When considering these distributions one may be wondering how the cut-off diameter $d_{c}$ is determined. The study of Driessen et al. [3] offers some insight. Indeed they show that ligament segments having aspect ratio $l / r_{0}>\Lambda_{c}$, where $\Lambda_{c}=I_{c} / r_{0}$ is a critical aspect ratio given below, will break before they finish retracting. The critical length is given by

$$
\frac{\left\|\log \left(\eta^{\prime}\right)\right\|}{t_{\sigma} \cdot \omega_{\max }}+\left(3 \Lambda_{c}\right)^{1 / 3}-\frac{\Lambda_{c}}{2}=0
$$

where $\eta^{\prime}$ is the noise amplitude in the ligament segment at the time of its formation, the capillary time scale is $t_{\sigma}=\left(\rho r_{0}^{3} / \sigma\right)^{-1 / 2}$ and the optimal growth rate $\omega_{\max }$ is a (weak) function of the Ohnesorge number, and is calculated from the dispersion relation obtained by Weber [17]. Note that $\eta$ ' is larger than the noise amplitude $\eta$ at the start of the simulation since corrugations have time to grow everywhere.

And alternate view on maximum ligament size maybe taken from the work of Anthony et al. and Notz \& Basaran $[1,12]$. There the maximum aspect ratio for $\mathrm{Oh}=10^{-3}$ is determined to be $\Lambda_{\mathrm{c} 2}=$ $7,83 \pm 0.5$, above which ligaments break by the end pinching mechanism. The equivalent dimensionless droplet size corresponding to this aspect ratio is $d_{d} /\left(2 r_{0)}=\left(3 \Lambda_{c 2} / 4\right)^{1 / 3}\right.$ which yields $d_{c} /\left(2 r_{0}\right)=1.8$ which is incompatible with the characteristics of the observed distribution. One thus has to admit that even if relatively short ligaments breakup by end pinching, the remaining part of the ligament survives perhaps through the "avoidance of end pinching" mechanism of Hoepffner \& Paré [7].

\section{Conclusion}

An intensive study of the ligament breakup allowed us to obtain an ensemble of approximately $10^{5}$ droplets. The distribution is multimodal with a mode corresponding to satellite droplets, a primary mode corresponding to the Rayleigh-Taylor instability and additional modes that are compatible with coalescence of the droplets in the primary mode. A striking effect is the abrupt cut-off of the tail of the distribution, which should be the focus of future investigations.

Excluding the cutoff, the tail of the distribution is well described by several functional forms, with the Gamma distribution a good compromise between the best fit Log-Normal and Normal (Gaussian) distributions.

\section{Acknowledgements}

We acknowledge the funding of the ERC-ADV grant TRUFLOW, the ANR NANODROP grant funded by the Fondation de France, the PRACE Covid grant of computer time and the PRACE multiyear grant TRUFLOW. We thank Lydia Bourouiba for numerous discussions, Gareth Mc Kinley for providing the initial idea of this research and Stéphane Popinet for sharing his basilisk code.

\section{References}

[1] Anthony, C. R., Kamat, P. M., Harris, M. T., \& Basaran, O. A. (2019). Dynamics of contracting filaments. Physical Review Fluids, 4(9), 093601. 
[2] Balachandar, S., Zaleski, S., Soldati, A., Ahmadi, G., \& Bourouiba, L. (2020). Host-tohost airborne transmission as a multiphase flow problem for science-based social distance guidelines. Int. J. Multiphase Flow, 132, 103439.

[3] Driessen, T., R Jeurissen, H Wijshoff, F Toschi, D Lohse, Stability of viscous long liquid filaments. Phys. fluids 25, 062109 (2013).

[4] Fuster, D., Matas, J.P., Marty, S., Popinet, S., Hoepffner, J., Cartellier, A.H. and Zaleski, S., 2013. Instability regimes in the primary breakup region of planar coflowing sheets. Journal of Fluid Mechanics, 736, pp.150-176.

[5] Gorokhovski, M, and M. Herrmann. "Modeling primary atomization." Annu. Rev. Fluid Mech. 40 (2008): 343-366.

[6] Gorokhovski M, Saveliev V. 2003. Further analyses of Kolmogorov's model of breakup. Phys. Fluids 15:184-92

[7] Hoepffner, J., and G. Paré. "Recoil of a liquid filament: escape from pinch-off through creation of a vortex ring." J. Fluid Mech 734 (2013): 183-197.

[8] Kolmogorov A.N. 1941. On the log-normal distribution of particles sizes during breakup process. Dokl. Akad. Nauk. SSSR 31:99-101.

[9] Y. Ling, D. Fuster, S. Zaleski, and G. Tryggvason. Spray formation in a quasiplanar gasliquid mixing layer at moderate density ratios: A numerical closeup. Physical Review Fluids, 2(1):014005, 2017.

[10] Y. Ling, D. Fuster, G. Tryggvason, and S. Zaleski. A two-phase mixing layer between parallel gas and liquid streams: Multiphase turbulence statistics and influence of interfacial instability. Journal of Fluid Mechanics, 859:268-307, jan 2019.

[11] Lohse, D., \& Villermaux, E. (2020). Double threshold behavior for breakup of liquid sheets. Proceedings of the National Academy of Sciences, 117(32), 18912-18914. http://doi.org/10.1073/pnas.2011358117

[12] Notz, Patrick K., and Osman A. Basaran. "Dynamics and breakup of a contracting liquid filament." Journal of Fluid Mechanics 512 (2004): 223.

[13] Opfer, L., Roisman, I.V., Venzmer, J., Klostermann, M. and Tropea, C., 2014. Dropletair collision dynamics: Evolution of the film thickness. Physical Review E, 89(1), p.013023.

[14] Popinet, S. An accurate adaptive solver for surface-tension-driven interfacial flows. Journal of Computational Physics, 228(16):5838-5866, 2009.

[15] Scharfman, B. E., Techet, A. H., Bush, J. W. M., \& Bourouiba, L. (2015). Visualization of sneeze ejecta: steps of fluid fragmentation leading to respiratory droplets. Experiments in Fluids, 57(2), 1-9.

[16] Villermaux, E. (2020). Fragmentation versus cohesion. J. Fluid Mech, 898, P1.

[17] Weber, C. , Disintegration of liquid jets. Z. Angew. Math. Mech. 1, 136-159 (1931). 